\title{
Timelines to Cervical Cancer Diagnosis and Treatment at a Tertiary Hospital in Botswana
}

\author{
Mercy-Nkuba Nassali ${ }^{1,2}$ \\ Tadele Melese ${ }^{1,2}$ \\ Jamieson Modimowame ${ }^{1,2}$ \\ Badani Moreri-Ntshabele $e^{1,2}$ \\ 'Department of Obstetrics and \\ Gynaecology, Faculty of Medicine, \\ University of Botswana, Gaborone, \\ Botswana; ${ }^{2}$ Department of Obstetrics \\ and Gynaecology, Princess Marina \\ Hospital, Gaborone, Botswana
}

Purpose: To describe the timelines leading to presentation, diagnosis and definitive treatment among cervical cancer patients at a tertiary treatment center in Botswana.

Patients and Methods: This was a retrospective study that evaluated timelines to diagnosis and linkage to definitive treatment among cervical cancer patients in Botswana. Medical records of 149 patients admitted at Princess Marina Hospital (PMH) from 2012 to 2014 were reviewed from August 2016 to February 2017. Data collected included sociodemographics, stage of disease at presentation, symptom duration at presentation, diagnosis to definitive treatment interval and treatment outcomes on discharge. STATA 12 was used for data analysis. Frequencies and percentages were used to analyse and present the data. This paper is limited to the analysis of records with documented duration of symptoms, histology turnaround time and the diagnosis to treatment interval.

Results: The median duration of symptoms at presentation $(\mathrm{N}=80)$ was 120 days (range 1-1290). Women who were HIV seropositive, of secondary level education or higher, below 50 years and those with cervical cancer screening history reported shorter duration of symptoms at presentation. Median histopathology turnaround time $(\mathrm{N}=123)$ was 27 days (range 3-274), median diagnosis to definitive chemoradiation interval $(\mathrm{N}=81)$ was 89 days (range 16-305) while median waiting time for surgery $(\mathrm{N}=7)$ was 60 days (range 29-279). Overall, the patients' journey from the community to definitive treatment was about six months.

Conclusion: Delayed cervical cancer diagnosis and treatment is multifactorial and entails a complex interplay between patient health-seeking behavioural patterns, robustness of the patient referral and follow-up mechanisms, availability of prompt histopathology services and relay of results, and timely linkage to definitive care. Prioritization of strategies to address hurdles in all these aspects will not only reduce waiting times but also ensure timely management and improved outcomes among patients with cervical cancer.

Keywords: diagnosis, timelines, cervical cancer, Botswana

\section{Introduction}

Cervical cancer remains the leading cause of cancer-related mortality among women in Sub-Saharan Africa. ${ }^{1}$ While disease elimination is the utmost goal globally, providing timely treatment and palliative care for incident cases of invasive disease remains a critical component of every successful national cervical cancer control program. ${ }^{2}$ Early presentation, timely diagnosis and access to treatment are critical to optimize treatment outcomes and survival among cervical cancer patients.

Achieving this outcome, however, entails a purposive integration of several components of patient care such as health-seeking behavior as evidenced by early
Correspondence: Mercy-Nkuba Nassali Department of Obstetrics and Gynaecology, Faculty of Medicine, University of Botswana, Private Bag, 007।3, Gaborone, Botswana

Tel +26776058947

Fax +2673105979

Email nassalim2@gmail.com 
presentation, timely evaluation and referral as necessary, access to histopathology to confirm diagnosis, prompt relaying of results to patients, a well-organized system for linking patients to definitive treatment and a vigilant post-treatment surveillance system for monitoring short, as well as long-term treatment outcomes.

Despite a relatively high level of awareness of cervical cancer among women in Botswana, ${ }^{3}$ most patients still present late, with already locally advanced disease. ${ }^{4}$ The introduction of gynecological cancer patient care in a multidisciplinary clinic setting since 2015 at the Princess Marina tertiary treatment center significantly reduced the number of visits required and time intervals needed to access lifesaving radiotherapy treatment by more than half. ${ }^{5}$ However, specific timelines leading up to patient initial presentation, diagnosis and associated factors are not known. This information is critical for understanding the patient's journey to treatment as well as for identifying gaps and barriers that need to be addressed to optimize patient outcomes. In this paper, we describe the timelines leading to presentation, diagnosis and definitive treatment among cervical cancer patients at a tertiary treatment center in Botswana.

\section{Patients and Methods Study Design}

This study was nested in a retrospective cross-sectional study that determined the predictors of locally advanced disease at presentation and clinical outcomes among cervical cancer patients in Botswana. Electronic and paperbased medical records of 149 cervical cancer patients previously admitted at Princess Marina Hospital (PMH) from May 2012 to December 2014 were reviewed from August 2016 to February 2017. ${ }^{4}$ Laboratory results are entered electronically while outpatient, inpatient, radiological and intraoperative clinical records are paper-based.

\section{Study Setting}

$\mathrm{PMH}$ is the national referral hospital for Southern Botswana, serving a catchment population of approximately 1.1 million people (52\% of the population). The Unit receives an average of 100-140 new cases of cervical cancer per year. Overall, gynecological malignancies accounted for $86 \%$ of the gynecology unit mortalities, and cervical cancer was the leading cause of death, accounting for $77 \%$ of gynecology-related mortality at PMH in 2019 (unpublished annual hospital reports). The facility mainly receives patients that are referred to it from lower health facilities, both public and private.

Histopathology services are offered by the National Health Laboratory (NHL), and the service is free of charge. Histology requests are submitted through the Integrated Patient Management System (IPMS) at hospitals, and specimens are delivered by hospital staff to the NHL, which is adjacent to PMH. Histology results are similarly posted in the IPMS. This ensures easy access by health workers at the tertiary center and across the country's public health sector system. Occasionally, a few patients referred from private facilities will have processed their histology in private laboratories. Definitive surgery is mainly offered at $\mathrm{PMH}$ and occasionally in private hospitals. Chemoradiation services are accessed at Gaborone Private Hospital (GPH), which is located approximately $2 \mathrm{~km}$ from PMH. An estimated 200 cervical cancer patients access radiotherapy per year at the unit. ${ }^{6}$ All diagnostic and treatment costs for nationals are covered by the government. Patient care and follow-up of patients referred to $\mathrm{PMH}$ directly from peripheral hospitals and through the daily Sexual Reproductive Health (SRH) clinics, well women health clinics and wards is coordinated at a dedicated weekly multidisciplinary gynecologic oncology clinic established in 2015. Prior to 2015, all evaluation and treatment decisions were taken at the SRH clinic. It is worth noting that in 2015 and 2016 the only radiotherapy unit in the country was closed for upgrade and patients eligible for this treatment were referred to several treatment centers in South Africa. There was limited tracking of progress and outcomes outside the country. As a result, enrollment for this study relied on available print and electronic records for patients admitted earlier through 2012-14.

\section{Sample Size}

Using the World Health Organization sample size calculator and based on the cervical cancer incidence in Botswana of $22 / 100,000$, the at-risk population of females over 25 years of age of 480,641 , a $95 \%$ confidence interval and a 5\% margin of error, a sample size of 108 was estimated and scaled up to 152 to cater for incomplete documentation. Details of sample size estimation are given in Nassali et al. ${ }^{4}$ Three incomplete entries were excluded. This paper is limited to the analysis of records with specific documentation of duration of symptoms $(\mathrm{N}=80)$, histology turnaround time $(\mathrm{N}=123)$ and the interval from diagnosis to definitive treatment modality $(\mathrm{N}=81)$. 


\section{Data Collection and Management}

A pretested data extraction form was used to abstract the data. Data collected included patient sociodemographics, stage of disease at presentation, duration of symptoms prior to presentation, histopathology turnaround time, timelines from diagnosis to definitive treatment as well as short-term patient outcomes on discharge.

The duration of symptoms in days was derived from the clinical records at the initial evaluation at PMH. Where multiple symptoms of variable durations were reported by the patient, the symptom with the longest duration was considered. Time of clinical diagnosis was taken as the time the patient had a cervical biopsy of the suspicious cervical lesion, which mainly occurred at the tertiary hospital. Occasionally, the diagnosis was made at a referring primary or district hospital. Histology turnaround time was captured from the IPMS, where each patient's report bears the date of receipt of the specimen by the laboratory and the date of final reporting and sign off. The date of definitive therapy was derived from either the intraoperative record for those patients who underwent surgery or the date of initiation of radiotherapy as noted in the GPH radiotherapy discharge record. A copy of this discharge record is given to the patient and another filed in the patients' inpatient records. The decision-to-treat to treatment interval was taken as the time from when a patient's histology results were reviewed at the SRH clinic, final staging and definitive plan finalized to when the treatment plan was actually effected. STATA 12 was used for data analysis. Frequencies were used to present the data. Analysis was limited to records that documented the respective timeframes.

\section{Ethical Approval}

Ethical approval to conduct this study was obtained from the respective ethical boards: the University of Botswana, the Botswana Ministry of Health and PMH (Permit number UBR/RES/IRB 1642). The research was conducted according to the principles of the Declaration of Helsinki. At the time of data collection, the patients had long been managed and discharged from the hospital. An institutional waiver of consent was given to access the patient's medical records. Confidentiality in handling of all medical records and patient data was maintained. Data collection was conducted at the hospital's records office during working hours and access was limited to the study team. No patient records were taken out and no patient identifier information was included in the retrieved data. A master admissions register for the study period was kept separately to aid retrieval of paper-based records by the records office and to access the IPMS.

\section{Results}

Chart retrieval rate was 152/261 (58.2\%) admitted cases from May 2012 to December 2014. Three incomplete entries were eliminated at initial data cleaning. Of the 149 patients, the response rate for symptom duration was 80/149 (53.7\%), initial consult to PMH referral interval 79/109 (72.5\%), histology turnaround time 123/149 (82.5\%), diagnosis to definitive treatment 91/149 (61\%), and decision-to-treat to treatment interval 35/91 (38.5\%).

The median duration of symptoms at presentation $(\mathrm{N}=$ 80) was four months. Mean referral time from lower units $(\mathrm{N}=79)$ upon suspicion of cervical cancer was 17 days. The median interval from cervical biopsy to availability of a posted histopathology report $(\mathrm{N}=123)$ in the IPMS was 27 days, and the median interval from availability of the report to patient review with results was $(\mathrm{N}=79) 19$ days. With regard to treatment interventions, 10 patients underwent curative surgery, 103 received chemoradiation ( 2 as adjuvant chemoradiation, 41 as radical chemoradiation with curative intent and 60 as palliative chemoradiation). Thirty patients were only eligible for supportive care, while six patients had no documented treatment intervention. Of the 113 receiving surgery and/or chemoradiation, only 91 records had specific documentation of timeframes. The median interval from clinical diagnosis to accessing chemoradiation ( $\mathrm{N} \mathrm{81/103)}$ was almost 3 months (89 days). Seven of the 10 patients who underwent surgery alone had a median diagnosis-to-surgery interval of 2 months (Table 1). Median decision-to-treat to treatment time was (N35/91) 36 days (range 3-274). Women of secondary level education or higher, HIV positive women, women below 50 years of age and those with a history of cervical cancer screening had a relatively short duration of symptoms at the time of presentation (Table 2). While a linear relationship between duration of symptoms and stage of disease at presentation may be anticipated, median duration of symptoms at presentation was surprisingly similar, irrespective of stage of disease and, paradoxically, lower with Stage 4 disease (Figure 1). 
Table I Timelines to Presentation, Diagnosis and Treatment Among Cervical Cancer Patients at a Tertiary Hospital in Botswana

\begin{tabular}{|l|r|l|l|l|l|}
\hline Variable & $\begin{array}{r}\text { Response } \\
\text { Rate }\end{array}$ & $\begin{array}{l}\text { Mean } \\
\text { (Days) }\end{array}$ & $\begin{array}{l}\text { SD } \\
\text { (Dedian } \\
\text { (Days) }\end{array}$ & $\begin{array}{l}\text { Range } \\
\text { (Days) }\end{array}$ \\
\hline Interval from onset of symptoms to presentation & $79 / 149$ & 187 & 17 & 285.67 & 120 \\
\hline Interval from suspected disease at initial facility to referral to PMH & $123 / 149$ & 38 & 54.84 & 1 & $1-2190$ \\
\hline Interval from cervical Biopsy to availability of report of results & $79 / 149$ & 30 & 44.99 & 19 & 27 \\
\hline $\begin{array}{l}\text { Interval from availability of histopathology report to patient review and } \\
\text { feedback }\end{array}$ & $81 / 103$ & 100 & 68.8 & 89 & $1-279$ \\
\hline Interval from clinical diagnosis to chemo radiotherapy & & 96 & 74.61 & 67 & $16-305$ \\
\hline Palliative RT N=45/8I & & 105 & 61.5 & 92 & $16-305$ \\
\hline Curative RT N=36/8I & $7 / 10$ & 125 & 110.15 & 60 & $30-251$ \\
\hline Clinical diagnosis to definitive surgery & & $29-279$ \\
\hline
\end{tabular}

\section{Discussion}

\section{Duration of Symptoms}

Mean duration of symptoms prior to presentation was 186 days (26.8 weeks), slightly higher than findings of a Malawi study of 23 weeks to presentation. ${ }^{7}$ Unlike the findings of the Malawi study, in which patients presenting with advanced disease (Stage 3 and 4) reported a duration of symptoms of almost a month longer than those presenting with an earlier stage of disease (1 and 2), our patients, at Stage 4, reported a shorter duration of symptoms compared to patients at earlier stages. This unexpected trend may be attributed to 1) patient recall bias or 2) unrecognized initial symptoms or underrating initial symptoms as insignificant. As a result, selfreported duration of symptoms may be limited to shorter timeframes when symptoms were deemed severe or unbearable due to emerging complications. Lim et al categorize symptoms of cervical cancer as 1) the initial symptom, 2) the symptom the patients could attribute to cervical cancer in retrospect, and 3) the trigger symptom. ${ }^{8}$ Patients often report the shorter interval that correlates with the onset of the trigger or life-threatening symptom. This may lead to an overall underestimation of symptom duration in clinical evaluation. On the other hand, a shorter duration could be reported to avoid blame. To cater for this observation, the symptom with the longest duration was considered when capturing symptom duration in our study.

A London-based study of symptomatic cervical cancer patients noted a mean trigger symptom duration of 1 month (range 0-4 months), ${ }^{8}$ a much shorter finding compared to our study, possibly highlighting better symptom recognition, better health-seeking behavior and referral patterns in higher income settings.

In a qualitative assessment of symptomatic women with cervical cancer in Uganda, Mwaka et al report that patient perceptions of themselves as being at low risk for disease led to delayed presentation because they considered their initial symptoms as either normal or attributable to more common infections such as sexually transmitted infections (STI). However, presentation was triggered when symptoms were unresponsive to self-medication or became life-threatening such as heavy vaginal bleeding. ${ }^{9}$

An estimated $95 \%$ of the Botswana population lives within an $8-\mathrm{km}$ radius of the nearest health facility, with $96 \%$ of the urban and $72 \%$ of the rural population living within a 5 -km radius, respectively. ${ }^{10}$ Reasons for late presentation despite relatively easy access to free health-care warrants further exploration to ensure targeted health education interventions and improved health-seeking behavior campaigns.

\section{Diagnosis to Definitive Treatment}

The mean interval from clinical diagnosis to accessing chemoradiation was approximately 100 days (SD 69). This finding is similar to the interval reported in South Africa, a setting with a similarly high demand for limited radiotherapy services, where mean diagnosis-toradiotherapy waiting time among cervical cancer patients was 119 days. $^{11}$ Our median clinical diagnosis-toradiotherapy initiation interval of 89 days was more than twice the median interval reported by Grover et al of 39 
Table 2 Patients' Sociodemographic and Clinical Characteristics and Their Comparative Duration of Symptoms at Presentation to Princess Marina Hospital

\begin{tabular}{|c|c|c|c|c|c|}
\hline Characteristics & Response Rate & Mean Duration of Symptoms & Median & Range & SD \\
\hline \multicolumn{6}{|l|}{ Education level } \\
\hline Primary and below & $34 / 57$ & 246.14 & 135 & $1-2190$ & 399 \\
\hline Secondary and above & $21 / 50$ & 182.9 & 90 & $3-60$ & 179.3 \\
\hline Unknown & $25 / 42$ & 108.32 & 60 & $7-365$ & 107.28 \\
\hline \multicolumn{6}{|l|}{ Employment } \\
\hline Employed & $19 / 37$ & 183.7 & 150 & $3-600$ & 170.2 \\
\hline Unemployed & $59 / 108$ & 190 & 90 & $1-2190$ & 318.7 \\
\hline Unknown & $2 / 4$ & 108.5 & 108.5 & $7-210$ & 143.5 \\
\hline \multicolumn{6}{|l|}{ Marital status } \\
\hline Married & $17 / 37$ & 138 & 90 & $2-365$ & 137.8 \\
\hline Unmarried $^{\mathrm{a}}$ & $59 / 105$ & 208.5 & 120 & $1-2190$ & 321.8 \\
\hline Unknown & $4 / 7$ & 67.5 & 45 & $30-150$ & 56.79 \\
\hline \multicolumn{6}{|l|}{ Age } \\
\hline$<50$ years & $39 / 86$ & 135.2 & 90 & $3-600$ & 142.39 \\
\hline$\geq 50$ years & $4 I / 63$ & 235 & 150 & $1-2190$ & 370 \\
\hline \multicolumn{6}{|l|}{ Address } \\
\hline Rural & $61 / 105$ & 193.5 & 120 & $2-2190$ & 317 \\
\hline Urban & $|8 / 4|$ & 152.5 & 90 & $1-365$ & 142.2 \\
\hline Unknown & $1 / 3$ & 365 & - & - & - \\
\hline \multicolumn{6}{|l|}{ Referral status } \\
\hline Documented referral from other facility & $55 / 109$ & 200 & 120 & $1-2190$ & 330.9 \\
\hline Index consultation at PMH (self- referral) & $22 / 34$ & 169.3 & 120 & $2-395$ & 149 \\
\hline No documentation of referral status (unknown) & $3 / 6$ & 60 & 60 & - & 0 \\
\hline \multicolumn{6}{|l|}{ Referral facility level $n=109 / 149$} \\
\hline Local clinic & $13 / 20$ & 121.69 & 42 & $1-600$ & 180.3 \\
\hline Primary Hospital & $24 / 44$ & 140.38 & 120 & $5-365$ & 125.32 \\
\hline District Hospital & $12 / 32$ & 444.7 & 270 & $30-2190$ & 618.4 \\
\hline Private clinic & $1 / 4$ & 7 & - & - & - \\
\hline Private Hospital & $0 / 1$ & - & - & - & - \\
\hline Other Tertiary Hospital (NRH) & $4 / 8$ & 138 & 144 & $90-180$ & 34.2 \\
\hline \multicolumn{6}{|l|}{ HIV Serostatus } \\
\hline Positive & $49 / 97$ & 165.06 & 90 & $1-2190$ & 319.12 \\
\hline Negative & $22 / 4 \mid$ & 248.7 & 210 & $5-1095$ & 239.17 \\
\hline Unknown & $9 / 11$ & 150.9 & 60 & $2-395$ & 170.7 \\
\hline \multicolumn{6}{|l|}{ Cervical cancer screening history in last 3 years } \\
\hline Yes & $32 / 57$ & 176.5 & 75 & $1-1095$ & 220.37 \\
\hline No & $44 / 77$ & 198.12 & 135 & $2-2190$ & 335.8 \\
\hline Unknown & $4 / 5$ & 138 & 90 & $7-365$ & 158.2 \\
\hline
\end{tabular}

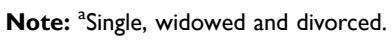

days from cervical biopsy to start of radiotherapy, following establishment of a gynecological cancer multidisciplinary team (Gyn MDT) at the PMH tertiary center (almost a year after the period of attendance of most of our cases) that streamlined linkage of care and reduced waiting times to treatment. ${ }^{5}$ The difference could also be explained by the higher proportion of inpatients with advanced disease (55\% with Stages 3 and 4) in our sample and the complications thereof, which often required longer prechemoradiation optimization and resulting in delayed 


\section{Median duration of symptoms and median diagnosis to intervention interval versus stage of disease at presentation}

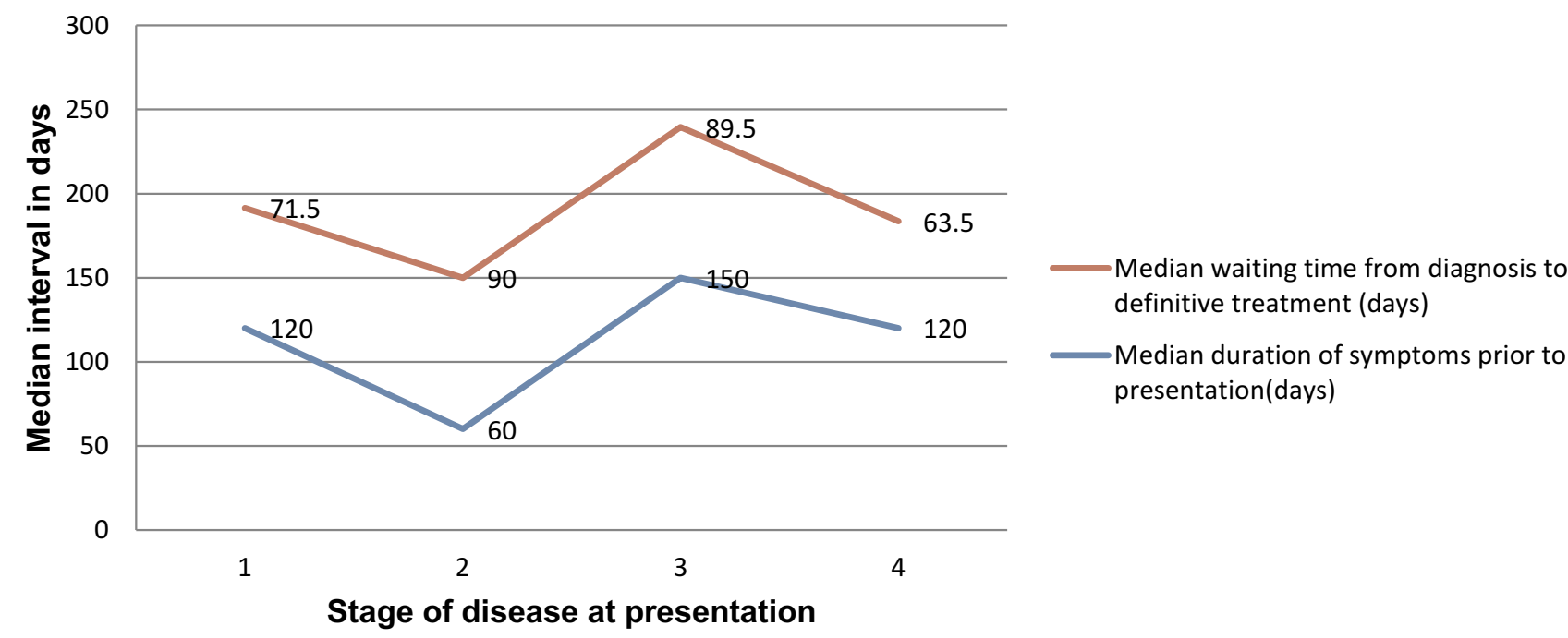

Figure I Median duration of symptoms at presentation and median diagnosis to intervention interval among cervical cancer patients admitted at Princess Marina Hospital, Botswana, 2012-2014.

initiation of definitive treatment in our study. ${ }^{4}$ Patients evaluated and treated as outpatients leave with all their records and were not included.

This diagnosis-to-definitive-treatment interval encompasses a complex interaction of various factors ranging from proper and timely patient evaluation at the various clinics and hospitals, efficient referral to $\mathrm{PMH}$, histology turnaround time, booking and adherence to follow-up visits where coordinated linkage to chemoradiation or surgical intervention is finalized, government health-care costcover guarantee endorsement for outsourced radiotherapy services, to one's medical fitness to initiate the assigned definitive therapy (Figure 2). The median waiting time from diagnosis to treatment of 89 days in our study still falls way below the international targets in high-income countries, such as the United Kingdom, of 62 days from general practitioner referral to start of definitive treatment, ${ }^{12}$ compared to the 4 week recommendation by the 2020 WHO strategy for scale-up of treatment for cervical cancer. ${ }^{13}$ Our mean 17 -day referral interval from initial local clinic or hospital to tertiary specialist evaluation was close to the 14-day recommendation by the England National Health System. ${ }^{14}$

Delayed initiation of a definitive intervention negatively impacts the patient and therapeutic outcomes, with effects ranging from prolonged anxiety and distress to disease progression requiring upstaging. Over a 10week waiting period, $43 \%$ of patients in a South African study showed evidence of disease progression. Our study, being retrospective, did not evaluate the impact of waiting time with regard to progression of the stage of disease. The mental health aspects of waiting times are also often underestimated. However, Song et al have described the mental health concerns among patients with longer waiting times, requiring psychiatric hospital care within the first year of treatment, with greater risk reported among those with previous history of a mental health disorder. ${ }^{15}$

\section{Biopsy to Availability of Results}

Botswana faces a high demand for the limited histopathology services. ${ }^{6}$ Our median diagnostic histopathology turnaround time of 4 weeks is higher than the 2 weeks recommended by $\mathrm{WHO}^{13}$ and the 7-day recommendation in United Kingdom. ${ }^{16}$ Long waiting times pose considerable anxiety for patients and family as they await the next steps. It took an additional 19 days in our study to relay results. Improved pathology capacity through training and better equipment is a critical rate-determining step. Consistent pathologist representation in the MDT tumor board is critical to facilitate availability of patients' results prior to their review appointments. Standardization of maximum turnaround times for suspected cancer specimens is critical, with clear mechanisms for the timely delivery of results. ${ }^{17}$ Digitalized laboratory notifications to the attending clinician and patients or their next of 


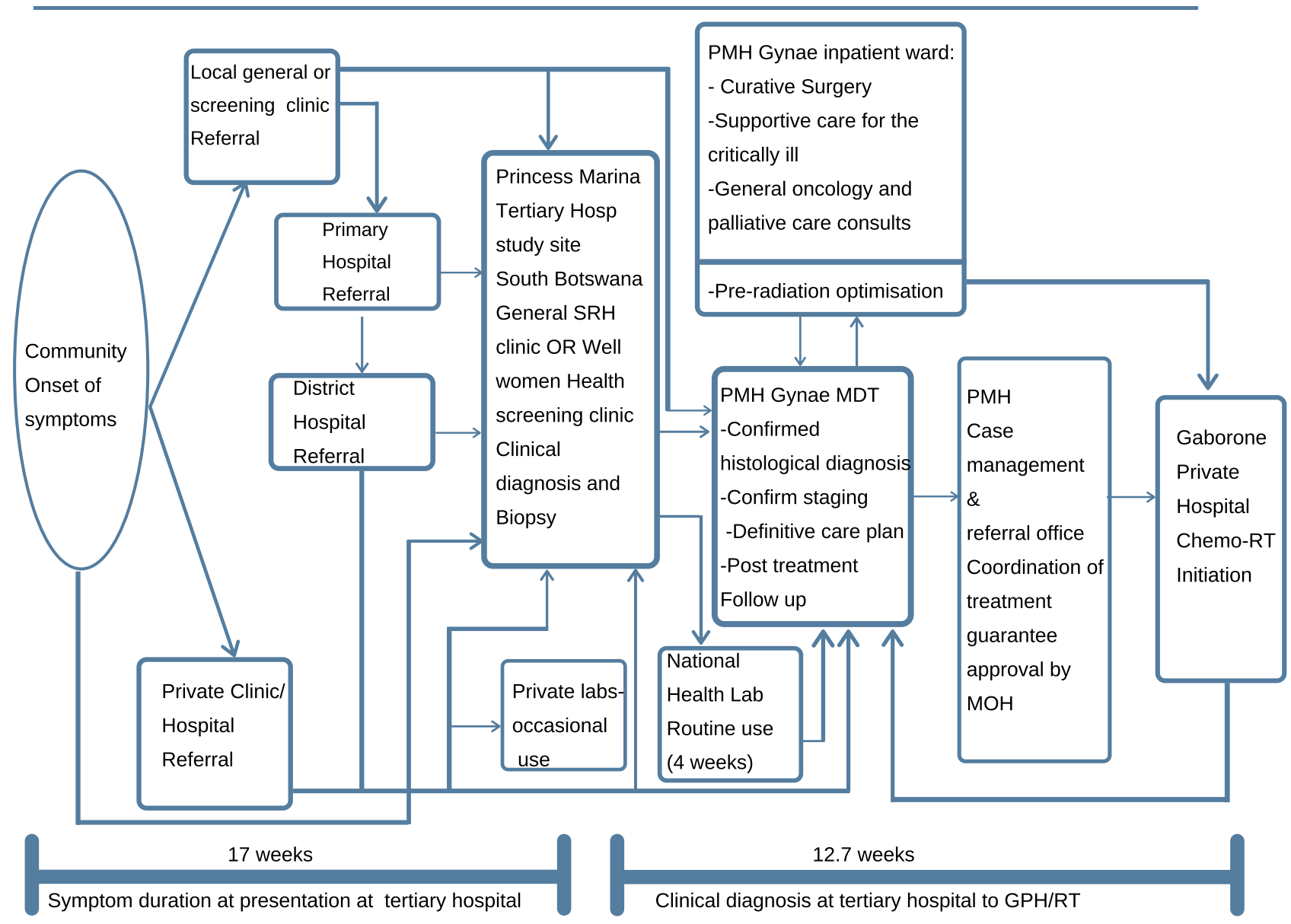

Figure 2 Cervical cancer patient's pathway to diagnosis and treatment at Princess Marina Hospital.

kin, as soon as results are published and ready for review, may further reduce delays.

\section{Access to Definitive Surgery}

Very few patients in our study were amenable to surgery. Of the $7 / 10$ with timelines to surgery, the median waiting time was 60 days. This finding is longer than that of an earlier study done in Botswana ${ }^{5}$ that reported a median time of 31 days from the decision to treat (at the MDT clinic visit) to surgery. Our study, however, considered the time span from initial clinical diagnosis (general gynecology specialist clinic at the tertiary hospital) to date of surgery. This interval incorporates the laboratory processing time as well, which made it inevitably longer. The sample size of seven surgical candidates was small, however, and may not be generalizable. Although five of these seven underwent surgery within 5 weeks, two outliers whose diagnosis of microinvasive disease followed a loop electrosurgical excision procedure (LEEP) for high-grade cervical intra epithelial lesion received their LEEP histology report 9 months and
1 year post-biopsy. They were then operated on in the following 2 months. However, the date of diagnosis was determined as the date the LEEP was reported, making the diagnosis to surgery interval longer. This highlights the need to prioritize LEEP histopathology results and close patient follow-up.

Definitive surgery referred only to simple hysterectomy for early microinvasive disease up to Stage 1A1 that was performed by general gynecology specialists due to lack of gynecologic oncology expertise at the time of the study. As a result, higher Stage 1 disease that could otherwise have been amenable to radical hysterectomy was managed with definitive chemoradiation, whose therapeutic outcome was deemed equivalent. This is in agreement with findings by SanGang Wu et al. ${ }^{18}$ Clinical practice at the PMH oncology unit has since changed, with more patients with early-stage disease accessing radical surgery as the primary intervention at shorter time intervals due to a weekly gynecologic oncology surgery service and following recruitment of a gynecologic oncologist in 2019. In 
Malawi, similar surgical delays were noted, with only $40.8 \%$ of those who needed surgery having been operated on by the end of 2 months following diagnosis. ${ }^{7}$ Limiting factors to timely access to surgery locally include availability of theater space and time due to competing emergency obstetric cases, blood shortages, as well as anesthesia service coverage shortages.

\section{Study Limitations}

Our findings of timelines to presentation, diagnosis and treatment offer baseline data for guiding nationally targeted interventions and monitoring improvement following their implementation. However, they ought to be interpreted in light of the following limitations. Although the retrospective medical records review offered details of a patient's journey through diagnosis and treatment, we were limited as regards assessing patient perspective about barriers to or factors facilitating timely presentation. Incomplete clinical documentation of specific timelines at various stages of patient care was a barrier to detailed analysis. The duration of symptoms may be affected by patient recall bias following the distress of diagnosis or a tendency to report a shorter duration relating to a trigger or life-threatening symptom. A standardized symptom duration classification tool as proposed by Lim et al should be considered ${ }^{8}$ in order to address inter-interviewer variation during history taking. The records reviewed were of previously admitted patients who may have been weaker, requiring longer times of pre-treatment optimization, or of those from further rural locations that required hospital lodging at $\mathrm{PMH}$ while on treatment at GPH. Both these categories could have contributed to longer intervals to definitive radiation as well as longer symptom duration. Nevertheless, this study provides important baseline information regarding the quality of care for cervical cancer patients.

\section{Future Prospects}

An in-depth exploration of patient and health-worker perspectives regarding delayed presentation and reasons for delayed diagnosis right from initial local clinics is worth undertaking for both inpatients and outpatients. Evaluation for possible disease progression, requiring upstaging, and mental health impact while awaiting definitive care should be considered in order to better understand the impact of waiting times. Standardized history-taking templates with regard to specific symptom duration may limit interclinician variations. Improved documentation will facilitate monitoring and evaluation of the impact of quality improvement interventions on therapeutic outcomes.

\section{Conclusion}

Achieving a timely diagnosis and treatment of cervical cancer patients entails a complex combination of patient healthseeking behaviour, proper patient evaluation and timely referral systems, access to efficient histopathology services, a well-organized multidisciplinary approach to definitive treatment, and a robust patient follow-up system. To ensure optimal outcomes, these critical components of care must be prioritised to reduce delays in accessing definitive care.

\section{Acknowledgments}

The authors are grateful to the staff at the Princess Marina Hospital who facilitated the access to the medical records and in data collection.

\section{Disclosure}

The authors report no conflicts of interest in this work.

\section{References}

1. Marc A, Elisabete W, Laia B, et al. Estimates of incidence and mortality of cervical cancer in 2018: a worldwide analysis. Lacent Global Health. 2020;8(2):E191-E203.

2. World Health Organization. Comprehensive Cervical Cancer Control: A Guide to Essential Practice. Second ed. 2014; 49-54.

3. Mingo AM, Panozzo CA, DiAngi YT, et al. Cervical cancer awareness and screening in Botswana. Int $J$ Gynecol Cancer. 2012;22 (4):638. doi:10.1097/IGC.0b013e318249470a

4. Nassali MN, Tadele MB, Robert MN, Jamieson M, Iwuh E, Katse E. Predictors of locally advanced disease at presentation and clinical outcomes among cervical cancer patients admitted at a tertiary hospital in Botswana. Int J Gynecol Cancer. 2018;28(6):1218-1225. doi:10.1097/IGC.0000000000001284

5. Grover S, Chiyapo S, Puri P, et al. Multidisciplinary gynecologic oncology clinic in Botswana: a model for multidisciplinary oncology care in low- and middle-income settings. J Glob Oncol. 2017;3 (5):666-670. doi:10.1200/JGO.2016.006353

6. Grover S, Raesima M, Bvochora-Nsingo M, et al. Cervical cancer in Botswana: current state and future steps for screening and treatment programs. Front Oncol. 2015;5:1-5. doi:10.3389/fonc.2015.00239

7. Rudd P, Gorman D, Meja S, et al. Cervical cancer in southern Malawi: a prospective analysis of presentation, management, and outcomes. Malawi Med J. 2017;29(2):124-129. doi:10.4314/mmj. v29i2.9

8. Lim AWW, Forbes LJL, Rosenthal AN, et al. Measuring the nature and duration of symptoms of cervical cancer in young women: developing an interview-based approach. BMC Women's Health. 2013;13:45. doi:10.1186/1472-6874-13-45

9. Mwaka AD, Okello ES, Wabinga H, Walter FM. Symptomatic presentation with cervical cancer in Uganda: a qualitative study assessing the pathways to diagnosis in a low-income country. $B M C$ Women's Health. 2015;15:15. doi:10.1186/s12905-015-0167-4

10. Statistics Botswana. Health Statistics Brief 2007-2015. Gaborone: Statistics Botswana; 2017. 
11. Lohlun K, Kotzen J, Lakier R. A prospective study on the impact of waiting times for radiotherapy for cervical cancer at Charlotte Maxeke Johannesburg Academic Hospital, South Africa. S Afr J Obstet Gynaecol. 2015;21:6-9. doi:10.7196/ sajog. 985

12. Powis S. Clinically-led Review of NHS Access Standards. 2019.

13. World Health Organization. Framework for Strengthening and Scaling-Up Services for the Management of Invasive Cervical Cancer. Geneva: World Health Organization; 2020.

14. Di Girolamo C, Walters S, Gildea C, et al. Can we assess cancer waiting time targets with cancer survival? A population-based study of individually linked data from the National Cancer Waiting Times monitoring dataset in England, 2009-2013. PLoS One. 2018;13(8):e0201288-e0201288. doi:10.1371/journal.pone. 0201288
15. Song H, Fang F, Valdimarsdóttir U, et al. Waiting time for cancer treatment and mental health among patients with newly diagnosed esophageal or gastric cancer: a nationwide cohort study. $B M C$ Cancer. 2017;17(1):2. doi:10.1186/s12885-016-3013-7

16. Royal College of Pathologists. Key Performance Indicators in Pathology. Recommendations from the Royal College of Pathologists. 2013.

17. Singh H, Vij MS. Eight recommendations for policies for communicating abnormal test results. Joint Commission J Qual Patient Saf. 2010;36(5):226-AP222.

18. Wu SG, Zhang WW, He ZY, Sun JY, Wang Y, Zhou J. Comparison of survival outcomes between radical hysterectomy and definitive radiochemotherapy in stage IB1 and IIA1 cervical cancer. Cancer Manag Res. 2017;9:813-819. doi:10.2147/CMAR.S145926

\section{Publish your work in this journal}

The International Journal of Women's Health is an international, peerreviewed open-access journal publishing original research, reports, editorials, reviews and commentaries on all aspects of women's healthcare including gynecology, obstetrics, and breast cancer. The manuscript management system is completely online and includes a very quick and fair peer-review system, which is all easy to use. Visit http://www.dovepress.com/testimonials.php to read real quotes from published authors. 